\title{
Plaidoyer pour la traduction de la poésie à l'École Normale Supérieure au Gabon
}

\author{
Liliane Surprise OKOME ENGOUANG Ep. NZESSEU ${ }^{1}$
}

\begin{abstract}
In the educational world of Gabon, the poem is an accepted pedagogical support in the progress made by the National Pedagogical Institute. But in the three foreign language departments of the Ecole Normale Supérieure in general and in the Spanish Department in particular, the lack of translation of poetry as an educational activity is a fact which, until today, weakens the training and hence, the students-teachers leaving and in class situation. This essay, which focuses on interpretative, literary and linguistic theories, aims to understand the reasons behind this deficient situation, but above all to propose an exploitation of the poem A Dorila of Juan Meléndez Valdés. Il will be a question of exposing the path that leads to the translation of a Spanish poem and implicitly to highlight the contribution of this activity in the leaners.
\end{abstract}

Keywords: translation ; poetry ; Spanish ; ENS ; Gabon

DOI: $10.24818 / \mathrm{DLG} / 2021 / 38 / 14$

\section{Introduction}

$\mathrm{L}$ 'École Normale Supérieure (ENS) est une institution d'enseignement public créée par l'ordonnance $n^{\circ 59 / 71 ~ d u ~} 4$ octobre 1971, grâce à un partenariat avec le Canada. Par décret n 651/PR/MENJSCF/MESRS fixant ses principes de fonctionnement et ses cycles de formation et de perfectionnement, elle a pour missions régaliennes «la formation professionnelle et le perfectionnement des candidats aux emplois de l'Éducation nationale ", (Journal Officiel de la République Gabonaise, 1997, p. 130). L'ENS est composée de dix-sept départements pédagogiques dont trois sont commis pour l'enseignement des langues étrangères, à savoir : les départements d'anglais, d'allemand et d'espagnol. Dans la présente étude, nous nous intéressons particulièrement au département d'espagnol, lequel compte la traduction pédagogique comme discipline inscrite à son programme de formation.

${ }^{1}$ Liliane Surprise Okome Engouang Ep. Nzesseu, École Normale Supérieure, CRAAL, CRAHI, Libreville, Gabon, lokomeengouang@gmail.com 
En tant qu'enseignante de ladite école et précisément du département au cœur de notre préoccupation, nous remarquons que le poème comme support n'est exploité que lors des enseignements de littérature (espagnole et ou hispanoaméricaine). Autrement dit, il demeure déficient dans les activités de traduction pédagogique, les phrases et les autres textes en prose y étant des supports privilégiés. Nous pensons, cependant, que pour que l'enseignement de la traduction pédagogique développe, chez les apprenants, les compétences requises, il est nécessaire d'initier et d'habituer ces enseignés à tous types de textes inscrits dans les progressions de l'enseignement secondaire établies par l'IPN en République gabonaise. G. Steiner (2003, p. 26-27) souligne à juste titre que :

Enseigner sérieusement, c'est poser les mains sur ce qu'il y a de plus vital chez un être humain. C'est essayer d'accéder au plus vif et au plus intime de l'intégrité d'un enfant ou d'un adulte. [...] L'enseignement médiocre, la routine pédagogique, un style d'instruction qui, délibérément ou non, vise avec cynisme des objectifs simplement utilitaires, sont ruineux. Ils extirpent l'espoir à sa racine.

Le niveau d'instruction étant l'un des indicateurs par lesquels on détermine l'Indice de Développement Humain (IDH) d'un pays (M. Fleurbaey, D. Blanchet, 2013), l'éducation, c'est-à-dire la formation des individus, y apparaît comme un facteur indispensable. Cela signifie qu'au sortir de l'ENS, les élèves-professeurs doivent pouvoir et savoir exploiter les différents types de supports-textes dans la pratique de leur métier.

Quelques discussions effectuées avec des collègues de l'ENS ont permis de comprendre que les causes de l'absence du poème en classe de traduction didactique sont plurielles. D'abord, il y a «la complexité du texte ; la quasi impossibilité à se mettre dans la peau d'un poète ; la méprise de la démarche pour traduire un poème». Ensuite, «le manque d'enseignants spécialistes de la traduction dans ce département est un facteur qui empire cette situation ». Un regard sur les différentes théories littéraires nous permet de dire que cette situation de l'ENS n'est guère singulière, car depuis l'Antiquité il est reconnu que la traduction de la poésie présente des difficultés particulières (R. Ellrodt, 2006). Certains auteurs ont même déclaré cette activité impossible ou du moins qu'elle reste l'exclusivité des seuls poètes. Maurice Blanchot (cité par R. Ellrodt, 2006, p. 1) épouse bien cette thèse et écrit à ce propos que : «Le sens du 
poème est inséparable de tous les mots, de tous les mouvements, de tous les accents du poème. Il n'existe que dans cet ensemble et il disparaît dès $q^{\prime} u^{\prime}$ on cherche à le séparer de cette forme qu'il a reçue ». Pour sa part, Dante (cité par G. Mounin, 1955, p. 28) explique qu' « aucune chose de celles qui ont été mises en harmonie par lien de poésie ne peut se transporter de son idiome en un autre sans qu'on alterne sa douceur et son harmonie ». En d'autres termes, traduire un texte poétique amènerait à défaire ledit texte de sa substantifique moelle et de son apparat linguistique car ce que le poème notifie coïncide précisément avec ce qu'il est.

Une observation attentive de la vie courante permet de constater que les gens parviendraient à déterminer ou imaginer l'état d'âme du musicien ou du chanteur à partir du rythme de sa musique. C'est en cela qu'on reconnaîtrait que la musique douce suggèrerait l'amour, le chagrin, la complainte et la musique forte, la joie, le bonheur ou la violence et la haine...si l'on s'en tient à certaines cultures. Par ailleurs, la poésie convoquant une musique dans les mots (Yves Bonnefoy, 2002), les éléments rythmiques constituent les moyens d'expression du poète. Les différents sons du texte qui se dégagent à partir de la combinaison des phonèmes (vocaliques et consonantiques) constituent la «musicalité » de celui-ci, sa mélodie. Il revient alors au lecteur-traducteur de la décrypter afin d'appréhender l'état d'âme et/ou l'intention du locuteur-poète. L'appréhension de la psychologie du poète trouve son importance dans la mesure où le discours est une mise en exergue de ses émotions, de ses sentiments. Et, selon la théorie de l'Ecole de Paris, la traduction est une affaire de compréhension, condition sine qua non de l'opération.

Le texte poétique obéissant à une forme d'écriture complexe et particulière, l'enjeu de notre analyse est de questionner la possibilité de traduire le poème en classe universitaire de langue au Gabon. Il s'agit précisément de décrire le schéma explicatif qui permettrait de saisir le contenu d'un poème pour en permettre la traduction. Par ailleurs, le travail entrepris contribuera, implicitement, à dévoiler l'apport de la traduction du poème dans le processus d'enseignement /apprentissage de l'espagnol. Elle formule donc l'hypothèse qu'en plus de développer l'expression écrite, la traduction du poème constitue un outil enseignement / apprentissage de la versification et la linguistique et, pour le cas d'espèce, la métrique et linguistique espagnole. Pour rendre opératoire cette hypothèse, le poème espagnol A Dorila de Juan Meléndez Valdés (1754-1817) est traduit par nos soins. Inscrite dans une perspective interprétative $(M$. Lederer et $D$. 
Seleskovitch, 1981), l'analyse s'appuie sur les travaux de G. Leclercq (2002), des Pardo (2007) et ceux de J.-C. Gémar (1995). Elle interroge d'abord les cadres contextuel, conceptuel et théorique qui sous-tendent l'étude. Ensuite elle expose le cadre méthodologique et le cheminement des opérations effectuées. En fin, elle passe en revue les résultats et en discute les contours.

\section{Cadres contextuel, théorique et conceptuel de l'étude}

Cette partie ouvre sur le contexte normalien qui fonde cette réflexion et fait apprécier le cadre théorique et le champ sémantique du terme clé de l'article.

\subsection{Contexte normalien}

Comme nous l'avons souligné en exorde, l'objectif assigné à l'ENS est la formation des enseignants et des acteurs de l'éducation nationale. Auparavant structurée en deux cycles de formation CAPC $^{2}$ et CAPES$^{3}$, elle est aujourd'hui composée du cycle Licence (trois ans) et du cycle Master (deux ans) conformément au système LMD en vigueur au lendemain des états généraux de l'éducation du 17 au 18 mai 2010. Les admis au concours d'entrée à l'ENS bénéficient d'une formation de trois ans. Au sortir de celleci, ils ont l'obligation ${ }^{4} \mathrm{~d}^{\prime}$ exercer pendant au moins cinq ans et ce, conformément au dispositif du Ministère de la fonction publique. À l'issue des cinq années d'exercice, chaque enseignant peut à nouveau candidater pour le cycle de Master afin de devenir enseignant du second cycle. Pour la présente étude, nous nous intéressons particulièrement au cycle Licence. Ce choix part du fait que les enseignements de traduction dudit département sont exclusivement destinés aux trois niveaux de ce parcours: Licence 1 , Licence 2 et Licence 3.

En Licence 1, le cours est essentiellement théorique au semestre 1 et pratique au semestre 2. Les enseignements théoriques sont basés sur l'origine de la diversité des langues, la définition et la méthodologie de la traduction. Le cours pratique axé sur les phrases vise à l'apprentissage et

${ }^{2}$ Certificat d'Aptitude au Professorat du Collège.

${ }^{3}$ Certificat d'Aptitude au Professorat de l'Enseignement Secondaire.

${ }^{4}$ Par décision interne à l'Ecole, des passerelles (continuité directe au Master) ont été accordées aux meilleurs étudiants. 
au raffermissement des structures de grammaire. En Licence 2, le cours de traduction est exclusivement de type pratique et porte tant sur des textes pragmatiques (pour des besoins en terminologie) que sur des phrases (à visée grammaticale). En Licence 3, par contre, l'activité est pratique mais, en règle générale, elle ne s'appuie que sur des supports littéraires. Ce choix d'exploiter le texte littéraire à ce stade de la formation n'est pas fortuit. En effet, le niveau pédagogique étant assez élevé, les apprenants peuvent désormais apprendre l'historicité des langues d'étude, certaines structures complexes et subtilités pour une meilleure manipulation et maitrise du mécanisme de fonctionnement de celles-ci. Les supports privilégiés ici ont presque toujours été des extraits de textes narratifs et les expressions idiomatiques. Aucun poème n'y est exploité malgré la richesse littéraire et linguistique qu'il renferme. Et au cours de certains débats de classe (enseignant-apprenants), les apprenants ont signifié, à l'unanimité, ne s'être jamais exercés à la traduction d'un poème depuis le début de leur formation. Cette situation justifie, encore une fois, les raisons qui motivent la présente étude.

\subsubsection{Approche théorique et conceptuel}

La présente étude relève de la traduction de la poésie telle que développée, entre autres, par G. Leclercq (2001, p. 86) et dont le résonnement corrobore celui de la théorie du sens de $M$. Lederer et D. Selescovitch (1981). Cette dernière estime que toute traduction de texte exige une démarche herméneutique ou exégèse de la part du traducteur. La traduction ne bénéficie pas d'une exhaustivité définitionnelle et encore moins fonctionnelle. En effet, des définitions lui sont commises conformément au contexte de sa pratique. Si dans un contexte professionnel la traduction s'inscrit dans un acte de communication inter ou intra linguistique, dans un contexte pédagogique elle constitue un outil d'enseignement / apprentissage des savoirs. Toutefois, quel qu'en soit l'objectif assigné à la discipline, son opérabilité est progressive. C'est pourquoi, ici, nous retenons la définition de l'École de Paris, selon laquelle, la traduction est un processus qui repose sur trois étapes: comprendre, déverbaliser et reverbaliser (M. Lederer et D. Seleskovitch, 1981).

La poésie du grec poiesis peut être définie comme un genre littéraire, à l'instar du théâtre et du roman, qui privilégie l'expressivité de la forme. $C^{\prime}$ est un art qui consiste à exprimer les sentiments et les idées au moyen 
d'un style et rythme particulier. Mais tout comme la traduction, elle ne jouit d'aucune exhaustivité définitionnelle tant elle recouvre une pratique diversifiée. En effet, d'après le dictionnaire de français Larousse, la poésie est «un ouvrage en vers, d'une certaine étendue ; un livret d'un opéra, $d^{\prime}$ 'une pièce lyrique ou une œuvre $d^{\prime}$ art ... qui suscite une émotion ». D'une façon assez simple, on peut dire que le poème, pièce de la poésie, est un texte qui combine les rythmes, les mots d'une langue pour évoquer des images ou suggérer des émotions. En effet, il y a la forme diversifiée du discours en relation avec la traduction, c'est-à-dire les caractéristiques qui définissent son mode d'expression en lui donnant une valeur esthétique. Le poème obéit à une tradition littéraire dont les caractéristiques obéissent à un rituel qui s'inscrit dans une forme d'organisation du discours déterminée par son organisation formelle, le rythme, la métrique, les strophes et son contenu; et la prise en compte de sa syntaxe. Autrement dit, la forme du poème « est pleinement constitutive du sens, de sorte que l'extériorité des notions de forme et de fond est invalide» (G. Leclercq, 2001, p.85). Donc, la traduction du poème exige que l'on comprenne les relations qu'il y a entre les phonèmes, les rimes, les mots et aussi pour comprendre ce que les différentes figures de rhétoriques véhiculent comme message dans le texte. À ce propos, G. Leclercq (2001, p. 86) déclare que :

Si je "démonte " le texte, c'est pour observer ses différentes composantes dont chacune possède des traits spécifiques, qu'ils soient manifestes, suggérés ou codés. C'est aussi pour comprendre le rôle des éléments, d'abord dans leurs caractères propres, ensuite dans leurs relations avec les autres éléments dont l'ensemble et l'assemblage spécifique font le texte.

La compréhension est une étape cruciale dans le processus de la traduction mais chaque type de texte exige une démarche exégétique appropriée pour la saisie de son contenu informatif. C'est dans cette optique que G. Leclercq (2001) porte sa théorie. Cette dernière est en quelque sorte une proposition méthodologique de traduction du poème. Son objectif est d'essayer d'apporter des solutions aux problèmes qui se posent tant au niveau de la compréhension du poème qu'au niveau de la traduction. Autrement dit, il s'agit ici de montrer comment, par une lecture préalable du poème, on arrive à accéder à sa compréhension puis à sa reformulation. Dans les prochaines lignes on se propose de dévoiler comment la traduction du poème $A$ Dorila s'opère. 


\section{Démarche méthodologique}

L'approche qualitative ou interprétative (T. Karsenti, L. SavoieZack, 2004) est le cadre de référence choisi pour la présente étude. Elle s'appuie sur «l'observation et le matériel écrit» (T. Karsenti, L. SavoieZack, 2004, p. 133). La donnée qui sert de base à notre analyse est le poème A Dorila, extrait du recueil des poèmes Obras en verso I de Juan Meléndez Valdés (1754-1817). De façon générale, le recueil compte trois chapitres et plusieurs poèmes classés par sections (v., gr., Letrillas, Elegías). Le poème A Dorila apparaît dans le chapitre III intitulé Odas anacreónticas et y occupe la sixième place.

On ne peut apprécier tous les textes littéraires de la même manière. En effet, on ne saurait lire un texte narratif de la même façon qu'on serait tenté de lire un poème, car chacun d'entre eux obéit à une forme d'organisation bien déterminée. Si donc tous les éléments du texte poétique servent à construire le sens, on comprend alors que la forme et le fond du texte sont équivalents dans l'expression verbale qui met en écriture et transforme l'évènement qu'il décrit. Cette étude met à profit les travaux de G. Leclercq ainsi que ceux de J.-C. Gémar et des Pardo dont les démarches permettent d'analyser, de décortiquer le poème et d'en dégager le contenu informatif. Pour mener à bien ce travail, nous avons commencé par l'analyse du poème selon les orientations de G. Leclercq (2001) et les travaux des Pardo (2007). Cette analyse a porté sur les éléments métriques (rimes, pauses, vers, etc.). Ensuite, nous avons procédé à l'analyse du texte selon J.-C. Gémar (1995), c'est-à-dire axée sur les éléments linguistiques (syntaxe, style, etc.). Les lignes qui suivent exposent au mieux les analyses qui ont mené à la saisie du contenu dudit poème.

\subsection{Analyse du schéma rythmique}

Cette étude consiste à découvrir le mécanisme de fonctionnement des unités rythmiques qui accordent un rythme particulier au vers et ensuite à l'ensemble du poème. A. Quilis (1999) précise que le schéma rythmique du texte est établi à travers l'étude du mètre, des pauses, de la ponctuation et de la rime.

\subsubsection{Le mètre}

L'analyse du mètre favorise la compréhension des vers que le poète donne à lire et à écouter. Pour réaliser cette étude, nous nous servons du 
modèle d'analyse des Pardo (2007) qui s'appuie sur les différentes composantes de la mesure du vers, c'est-à-dire le mètre, le rythme et la rime. Pour comprendre le rythme, une procédure est proposée par les Pardo (2007, p. 10) qui suggèrent que :

Pour mesurer le vers espagnol, c'est-à-dire pour établir le nombre de ses syllabes métriques, qui n'est pas forcément égal au nombre des syllabes phoniques, il faut : $1^{\circ}$ procéder au découpage du vers en unités syllabiques phoniques ; $2^{\circ}$ prendre en compte différents phénomènes tels que la synalèphe, la diérèse, etc., qui peuvent modifier le nombre des unités syllabiques; $3^{\circ}$ repérer le dernier accent tonique du vers et le numéro d'ordre de la dernière syllabe accentuée.

Si on s'en tient à ce qui vient d'être dit, la mesure du poème aide à comprendre la valeur du rythme dans le poème et elle est effectuée selon le modèle présenté dans les prochaines lignes.

\subsubsection{Les syllabes phoniques}

Pour la mesure du mètre, les Pardo (2007, p. 11) conseillent de commencer par découper le vers en syllabes phoniques car elles constituent la base de la mesure. En effet, ce sont elles qui permettent de réaliser une prononciation correcte de la langue, c'est pourquoi elles sont naturellement un fait de langue. Le tableau ci-dessous sert d'illustration :

\section{Tableau $1^{5}$ : Structuration du poème $A$ Dorila en syllabes phoniques}

\begin{tabular}{|c|c|}
\hline $\begin{array}{l}\text { S1 ¡Có-mo- se -van -las -ho-ras, } \\
\text { y- tras e-llas -los -dí-as, } \\
\text { y- los -flo-ri-dos- a-ños } \\
\text { de -nues-tra -frá-gil -vi-da! }\end{array}$ & $\begin{array}{l}\text { S5 Si -es-to, -pues, -nos- a-guar-da, } \\
\text { ¿pa-ra -qué,- mi -Do-ri-la, } \\
\text { son -los-flo-ri-dos- a-ños } \\
\text { de -nues-tra -frá-gil -vi-da? }\end{array}$ \\
\hline $\begin{array}{l}\text { S2 La -ve-jez -lue-go -vie-ne, } \\
\text { del -a-mor -e-ne-mi-ga, } \\
\text { y -en-tre -fú-ne-bres -som-bras } \\
\text { la -muer-te -se -a-ve-ci-na, }\end{array}$ & $\begin{array}{l}\text { S6 Pa-ra -jue-gos -y -bai-les } \\
\text { y -can-ta-res -y -ri-sas } \\
\text { nos -los -die-ron -los -cie-los, } \\
\text { las -gra-cias -los -des-ti-nan. }\end{array}$ \\
\hline $\begin{array}{l}\text { S3 que -es-cua-li-da -y -tem-blan-do, } \\
\text { fe-a,-in-for-me, -a-ma-ri-lla, } \\
\text { nos -a-te-rra, -y a-pa-ga } \\
\text { nues-tros fue-gos -y -di-chas. }\end{array}$ & $\begin{array}{l}\text { S7 Ven -jay! -¿qué - te- de-tie-nes? } \\
\text { Ven, -ven, -pa-lo-ma -mí-a, } \\
\text { de-ba-jo de -es-tas -pa-rras } \\
\text { do -le-ne -el vien-to -as-pira, }\end{array}$ \\
\hline
\end{tabular}

${ }^{5}$ Pour plus de concision, nous adoptons les abréviations suivantes : S1, S2 ... (première strophe, deuxième, etc.), V1, V2 ... (premier vers, deuxième vers...). 


\begin{tabular}{|l|l|}
\hline & \\
\hline S4 El -cuer-po-se-en-tor-pe-ce, & S8 y- en-tre -brin-dis -sü-a-ves \\
los -a-yes -nos- fa-ti-gan, & y -mi-mo-sas -de-li-cias \\
nos -hu-yen -los -pla-ce-res & de- la -ni-ñez -go-ce-mos, \\
y -de-ja la -a-le-grí-a. & pues -vue-la -tan -a-pri-sa. \\
\hline
\end{tabular}

Source : Données d'étude de septembre 2020

Le texte tel qu'il a été découpé révèle pour l'instant un fait naturel de la langue espagnole. Il y a également le phénomène métrique dont la lecture et la valeur sont examinées à travers la synalèphe, la synérèse, et la dernière syllabe accentuée.

\subsubsection{La synalèphe, la diérèse et dernière syllabe accentuée.}

La synalèphe est « la fusion, à l'intérieur d'un vers, en une seule syllabe métrique, de deux voyelles qui se trouvent en contact, l'une étant située à la fin d'un mot, et l'autre au début du mot suivant. Elles forment avec les consonnes respectives, une seule syllabe métrique » (Pardo, 2007, p. 12). Les cas de synalèphes sont présentés avec le trait "_" sous les voyelles concernées dont voici les exemples :

Tableau 2 : Illustration des synalèphes

\begin{tabular}{|l|l|l|}
\hline $\begin{array}{l}\text { S2 y_entre fúnebres sombras } \\
\text { la muerte se_avecina }\end{array}$ & $\begin{array}{l}\text { S4 el cuerpo } \\
\text { se_entorpece, } \\
\text { y deja la_alegría. }\end{array}$ & $\begin{array}{l}\text { S7 debajo de_estas } \\
\text { parras do lene_el } \\
\text { viento_aspira, }\end{array}$ \\
\hline $\begin{array}{l}\text { S3 que_escuálida_y } \\
\text { temblando, } \\
\text { fea,_informe,_amarilla, } \\
\text { nos aterra, y_apaga }\end{array}$ & $\begin{array}{l}\text { S5 si_esto, pues, nos } \\
\text { aguarda, }\end{array}$ & $\begin{array}{l}\text { S8 y_entre brindis } \\
\text { süaves }\end{array}$ \\
\hline
\end{tabular}

Source : Données d'étude de septembre 2020

La synalèphe n'influence en rien la prononciation du mot en langue espagnole, son impact se manifeste au niveau de l'organisation des syllabes métriques qui ne coïncident pas toujours avec les syllabes phoniques. La diérèse, quant à elle, c'est-à-dire, " séparation en deux syllabes métriques de deux voyelles d'une diphtongue » (M.\&A Pardo 2007, p.14) n'est présente que dans la strophe 8 v29 à travers le mot süave. En effet, elle transforme le mot süave en trisyllabe sü-a-ve à cause de l'accentuation du " $u$ ", la prononciation normale étant sua-ve. Cette diérèse serait un procédé 
technique qui sert à l'obtention d'un nombre de syllabes nécessaires au mètre et à donner un certain rythme au mot.

S'agissant finalement de la dernière syllabe accentuée, celle-ci porte l'accent métrique qui permet d'établir le mètre du vers selon qu'il se termine par, un mot oxyton (abrir $=$ a-brir $=2+1=3$ ) un mot proparoxyton (cántico $=$ can-ti-co=3-1=2) ou par un mot paroxyton (joven=jo-ven=1+1=2). L'analyse dévoile que tous les vers du présent texte sont paroxytons étant donné la position (pénultième) de leur accent final (S1 ¡Có-mo -se -van-las ho-ras; S2 y_en-tre fú-ne-bres som-bras) ${ }^{6}$. Aussi, notons-nous que le poème est constitué entièrement de vers heptasyllabes ${ }^{7}$, ce qui nous amène à conclure que le présent poème est de arte menor. Il appartient au groupe poétique ode anacréontique dont la principale caractéristique consiste à chanter avec une gracieuse légèreté les plaisirs de l'amour et de la table (Rozas, 2007).

\subsubsection{Les pauses}

Les pauses sont des silences, des arrêts ou des coupes qui répondent à des nécessités respiratoires, syntaxiques ou rythmiques (M. \& A. Pardo, 2007). Elles sont situées aux deux extrémités du vers qu'elles encadrent, ou à l'intérieur de celui-ci. Autrement dit, en plus de présenter un intérêt sémiotique, les pauses permettent d'analyser le rythme dans sa fonction esthétique et syntaxique. L'analyse prend en compte les différents types de pauses qui marquent à la fois la syntaxe et le mètre. Le présent poème dispose de pauses de fin de vers et de pauses de fin de strophes. Dans les pauses de fin de vers, on remarque des cas d'enjambement dit encabalgamiento versal dans quelques strophes par le signe de la pause de fin de vers entre parenthèses (S1 y los floridos años (//) de nuestra frágil vida// S3 nos aterra, y apaga (//) nuestros fuegos y dichas. S5 son los floridos años (//) de nuestra frágil vida ?). On constate qu'il s'agit, pour ces cas précis, d'encabalgamiento sirremático, car " il sépare une unité syntaxique intermédiaire entre le mot et la phrase qui, de par son fonctionnement à l'intérieur de la langue ne peut être scindé par une pause ». La construction normale des phrases S1 et S5 aurait pu être par exemple : ... y los floridos años de nuestra frágil vida. S3 : nos aterra, y apaga nuestros fuegos y dichas. Ces

${ }^{6}$ Par souci de concision, seules les deux premières strophes sont présentées.

${ }^{7}$ L'heptasyllabe fut l'un des vers les plus utilisés durant le Néoclassicisme dans les odas, romances, letrillas, endechas, anacreónticas, etc., (A. Quilis, 1999, p. 64). 
enjambements trouvent leur signification dans l'intention stylistique soulignée par la construction en hyperbate. Cette figure de style affecte le système de la langue en subvertissant la syntaxe au profit d'un effet poétique. En dehors du rôle que jouent les pauses métriques dans la lecture du poème, un autre regard est jeté sur la ponctuation.

\subsubsection{La ponctuation}

L'analyse de la ponctuation permet de lire la valeur des signes qui sont repartis dans le texte. Les signes pausaux sont caractérisés dans le texte par la virgule, le point, le point d'exclamation et le point d'interrogation. Dans cette étude, nous nous intéressons uniquement aux points d'exclamation et d'interrogation car nous pensons qu'ils sont les seuls à avoir vraiment une influence sur la traduction que nous proposons.

Dans le poème le point d'exclamation est présent deux fois. D'abord, il est utilisé dans la strophe 1, ensuite il est utilisé dans le vers 1 de la strophe 7. Placé au début de la strophe 1, il précède l'adverbe «como». Normalement cet adverbe relatif devient interrogatif lorsqu'il porte un tilde et est encadré par les points d'interrogation. Dans ce cadre il se traduit en français par «comment? » introduisant ainsi une question. Dans le poème, la présence du point d'exclamation (¡cómo!) rend finalement l'adverbe « exclamatif » car il exprime la qualité ou l'intensité du Temps à travers le verbe "passer" et nous amène à le traduire par «comme! ». Dans le vers 1 de la strophe 7 , l'exclamation marque la présence de l'interjection jay! qui exprime un sentiment de douleur et/ ou de tristesse. Dans un cas comme dans l'autre, on peut dire que ces exclamations marquent la tension émotionnelle du locuteur qui est davantage accentuée avec la présence des interrogations. En effet, le point d'interrogation apparaît deux fois dans le texte en S5 et en S7. Non seulement ces interrogations suggèrent la présence d'un locuteur mais également elles laissent entendre sa voix qui manifeste une préoccupation en ce sens qu'il se rendrait en évidence de la fugacité du temps, de la vie, sa faiblesse en tant qu'Homme et la douleur que cela procure.

La ponctuation ne semble pas complexe dans ce poème. Elle a une fonction rythmique qui participe à l'organisation syntaxique du texte, qui est liée à la production d'effets sémantiques. 


\subsubsection{La rime}

La musicalité étant le trait ornemental par lequel on définit la poésie, la rime est identifiée afin de souligner la marque d'oralité que porte le poème. Cette valeur renforce l'émotion et fait le lien entre le son et le sens du poème. En espagnol, il existe deux types de rime : la rima asonante (seuls les sons vocaliques sont pris en compte à partir de la dernière voyelle accentuée) et la rima consonante (c'est le retour sur deux ou plusieurs vers des mêmes phonèmes aussi bien vocaliques que consonantiques). Pour ce poème, la structure des rimes est identique dans toutes les strophes. En effet, pour chaque strophe, on note un certain retour des mêmes sons vocaliques dans les vers 2 et 4 . Autrement dit, dans chaque strophe, les vers pairs 2 et 4 ont une assonance grave en í-a et les vers impairs 1 et 3 restent dépareillés (sueltos). Chaque strophe obéit ainsi au schéma 7x-7a-7x$7 a$, comme indiqué dans le tableau 3.

Tableau 3 : Présentation des syllabes

\begin{tabular}{|l|l|l|l|}
\hline $\begin{array}{l}\text { horas }=\mathrm{x} \\
\text { dias }=\mathrm{a} \\
\text { años }=\mathrm{x} \\
\text { vida }=\mathrm{a}\end{array}$ & $\begin{array}{l}\text { viene }=x \\
\text { enemiga }=a \\
\text { sombras }=x \\
\text { avecina }=a\end{array}$ & $\begin{array}{l}\text { Temblando }=x \\
\text { Amarilla }=a \\
\text { Apaga }=x \\
\text { Dichas }=a\end{array}$ & $\begin{array}{l}\text { Entorpece }=x \\
\text { Fatigan }=a \\
\text { Placeres }=x \\
\text { Alegrí }=a\end{array}$ \\
\hline $\begin{array}{l}\text { Aguarda }=x \\
\text { Dorila }=a\end{array}$ & $\begin{array}{l}\text { Bailes }=x \\
\text { Años }=x\end{array}$ \\
Ridas $=a$ & $\begin{array}{l}\text { Cielos }=x \\
\text { Destinan }=a\end{array}$ & $\begin{array}{l}\text { Detienes }=x \\
\text { Mía }=a \\
\text { Parras }=x \\
\text { Aspira }=a\end{array}$ & $\begin{array}{l}\text { suaves }=x \\
\text { delicias }=a \\
\text { gocemos }=x \\
\text { aprisa } a\end{array}$ \\
\hline
\end{tabular}

Source : Données d'étude de septembre 2020

Quant à la rime assonante en $i-a$, elle confère une interjection répétitive tout au long du poème qui produit une mélodie mélancolique au texte, soit -ía-ía -ía-ía -ía-ía -ía-ía -ía-ía -ía-ía -ía-ía -ía-ía. L'interjection est entendue ici comme ce mot qui tient lieu d'une phrase qui traduit une émotion, un état intérieur. Nous pensons que ce son í-a qui détermine la rime du poème renvoie à un certain sentiment d'incapacité de la voix poétique à endiguer le cours irréversible du Temps; ce Temps qui dégrade physiquement et moralement l'être humain par l'arrivée de la vieillesse. En accord avec M. Lederer et D. Seleskovitch (1981) quant à leur méthode interprétative, J.-C. Gémar (1995) postule que pour que le sens d'un texte 
soit dégagé le plus justement possible, son lecteur-traducteur doit également procéder à une analyse de celui-ci sur les plans linguistiques.

\subsection{Analyse linguistique}

Dégager ce qu'évoque un texte revient à observer l'ensemble de l'organisation grammaticale et sémantique qui permet de lire ce qui lui donne sa cohésion (L. S. Okome Engouang, 2013). De la sorte, l'analyse linguistique que nous réalisons dans ces lignes est essentiellement basée sur les constituants syntaxiques et figures de rhétoriques.

\subsubsection{Les constituants syntaxiques}

Le but ici est d'identifier et d'interpréter les constituants syntaxiques du texte. On essayera d'expliquer la prédominance de certaines catégories grammaticales du poème tels que les verbes, déterminants, adjectifs et pronoms.

\subsubsection{Le verbe}

$\mathrm{Du}$ latin verbum, le verbe est un constituant important dans la phrase. Il est la portion du discours qui désigne l'existence, l'action ou passion des individus ou des choses, avec diverses terminaisons, de temps, de nombre et de personnes. L'analyse du verbe dans ce texte nous amène à considérer, d'une part, la valeur du temps verbal et, d'autre part, elle nous permet de déterminer la valeur du référent auquel ces verbes renvoient. Le texte compte un total de vingt (20) verbes dont quatorze (14) sont au présent de l'indicatif à valeur atemporelle et quatre (4) à l'impératif présent. S'agissant d'abord du présent de l'indicatif, il est réparti dans tout le texte, dans toutes les strophes : S1 (se van), S2 (viene, se avecina), S3 (aterra, apaga), S4 (se entorpece, fatigan, huyen), S5 (guarda, son), S6 (destinan), S7 (detienes, lene), S8 (vuela). Le présent de l'indicatif étant un temps de la narration, de vérité générale, sa forte représentation dans le texte trouve son explication dans l'intention de l'auteur d'actualiser le procès ou les faits, au sens où le discours suppose un destinataire.

Dans le poème, les verbes se van, viene, avecina, aterra, apaga, entorpece, fatigan, décrivent une action qui va de son commencement à son achèvement. Tout cela met en exergue l'action du Temps (infra, 2.2.2). Quant à l'impératif présent manifeste par : S7 (ven, ven, ven), S8 (gocemos), il évoque un procès contemporain de l'énonciation et ces verbes dévoilent le caractère hédoniste du discours. Aussi, faut-il apprécier la terminaison 
de gocemos. C'est la marque de la première personne du pluriel, elle suppose ainsi l'implication (omniprésence) du locuteur dans son discours et la présence d'un destinataire virtuel. Les deux temps présents font entendre la voix du poète (yo) hic et nunc, au moment où il parle, s'adressant ainsi au destinataire tú, Dorila, Paloma, c'est-à-dire tout lecteur.

\subsubsection{Les déterminants : adjectifs et pronoms}

L'analyse des déterminants permet d'actualiser le nom et de désigner un référent particulier auquel renvoie le locuteur. Ce poème comprend treize articles, tous étant définis et ils marquent plus la valeur des substantifs abstraits, tel S1 (las horas, los días, los años) S2 (la vejez), S4 (los placeres, la alegría), S6 (las gracias) S7 (el viento), S8 (la niñez). On peut dire que la présence de ces articles a un lien avec la focalisation interne du texte qui est aussi déterminée par la marque des adjectifs.

Le poème compte deux catégories d'adjectifs : les adjectifs possessifs et les adjectifs qualificatifs. Les premiers (nuestra (S1, S5), nuestros (S3), permettent d'établir la relation entre le terme déterminé et le possesseur (locuteur et destinataire). En d'autres termes, ces adjectifs possessifs jouent un rôle nominal, ils nomment directement le locuteur et le destinataire par la marque de la première personne du pluriel. Cela confirme la focalisation interne qui a été auparavant déterminée. Les seconds, pour leur part, sont tous épithètes ${ }^{8}$ (S1 (floridos años, frágil vida), S2 (fúnebres sombras), S3 (fea, informe, amarilla) et précèdent tous les noms qu'ils qualifient. Autrement dit, il s'agit ici des épithètes $\mathrm{d}^{\prime}$ ornement ${ }^{20}$, elles mettent en lumière les connotations possibles des noms qu'elles qualifient. Nous pouvons dire que ces adjectifs peignent le caractère destructeur de la vieillesse sur la jeunesse.

Le présent poème n'est pas très riche en pronom. On compte juste une récurrence du pronom personnel complément "nos" en S3v3, S4v2-3, $\mathrm{S} 5 \mathrm{v} 1, \mathrm{~S} 6 \mathrm{v} 3$ et le pronom complément " $t e^{\prime \prime}$ est présent une seule fois. Le pronom "nos" est la marque de la première personne du pluriel, il dévoile une fois de plus la présence du poète dans le texte, s'adressant à " $t e^{\prime \prime}$ qui suppose, toi, Dorila, paloma, qui renvoie à un destinataire virtuel. L'étude de l'organisation syntaxique des mots dans un texte permet de dégager la valeur stylistique qui a été déterminée. L'analyse des mots, quant à elle, est partie intégrante de l'étude sur la rhétorique.

${ }^{8}$ Infra 2.2.2. 


\subsubsection{Les figures de style}

L'analyse des figures de style consiste à apprécier les moyens d'expression du poète. Il s'agit dans ce cas précis de déterminer la valeur des images employées par le poète dans le souci de donner de la forme et du sens à son discours.

L'auteur introduit son texte par une gradation ascendante en S1 par les mots horas, días, años et vida, suivant un certain ordre croissant. On peut dire que cette figure met en évidence la notion de temps. Le Temps est en perpétuel mouvement, en évolution, il n'est pas statique, il passe. Le temps, compris ici comme l'existence de l'être, commence dès qu'on vient au monde et s'achève par la mort. Par cette image, le poète laisse entendre sa préoccupation pour ce phénomène naturel dont l'Homme (être éphémère) est prisonnier car, quoi qu'il en soit, il finit toujours par subir ses effets (Temps). En outre, on note dans le poème la marque de l'anaphore à travers le " $y$ " constamment en position initiale des vers dans les strophes $1 ; 3 ; 4 ; 6$ et 8 ). On peut dire que cette figure a valeur d'énumération. Les «y » permettent d'harmoniser les arguments, cela relèverait du goût esthétique de l'auteur à agencer ses idées pour une meilleure expression. Une anaphore est également présente dans les deux premiers vers de S7 par le verbe conjugué ven et elle est doublée d'une épizeuxe en V2 de S7.

L'épithétisme est également présent dans le texte en S1, S2, S5 et S8 par les adjectifs qualificatifs épithètes (floridos años; frágil vida; fúnebres sombras; floridos años, frágil vida; brindis süaves y mimosas delicias). Ensuite, on note que ces adjectifs précèdent tous les noms qu'ils qualifient. Autrement dit, il s'agit ici des épithètes d'ornement (20), elles mettent en lumière les connotations possibles des noms qu'elles qualifient. Comme interprétation, nous pouvons dire que cela relève du goût esthétique du poète d'harmoniser sa rime. Et d'ailleurs, l'épithétisme « représentait l'une des marques d'écriture les plus soutenues en Espagne et elle a la particularité d'embellir le discours parce qu'elle est l'expression de quelque chose de saillant et de pittoresque », (P. Fontanier, 1968, p. 355).

En outre, nous notons la présence de l'énumération et de la métaphore dans S3 : (...) nos aterra y apaga nuestros fuegos y dichas. L'élément métaphorique serait dans ce cas fuegos qui désigne métaphoriquement flamme. L'auteur décrit l'action de la vieillesse sur l'Homme. La vieillesse est un phénomène qui met fin à la flamme ou aux plaisirs de l'être humain. Une seconde métaphore est mise en évidence dans la S7 : Ven, ven paloma mía et celle-ci est celle de l'aimée, elle est mise 
pour Dorila. Le terme colombe (paloma) compte parmi les universelles métaphores célébrant la femme (Chevalier et Cheerbrant, 1982). Le locuteur appelle colombe afin de mettre en relief non seulement ses sentiments amoureux mais aussi le plaisir que Dorila (la femme) procure, la douceur qu'elle dégage, sa beauté divine et la pureté qu'elle symbolise.

Enfin, nous notons la présence de la personnification du Temps à travers les deux éléments qui le caractérisent : la vie et la mort. Le Temps est personnifié à travers les verbes de mouvement en S1v1 se van, S2v1 viene, S2v4 se avecina. Ces verbes créent un personnage en mouvement en lui donnant un caractère humain à travers son action sur l'Homme. La mort, quant à elle, est personnifiée à travers les verbes de mouvement et les adjectifs qui la qualifient. Nous avons à cet effet en S2 v4 se avecina, S3 escualida, temblando, fea, informe, amarilla, aterra, apaga. Les différentes analyses réalisées sur le poème $A$ Dorila nous permettent, à présent, de faire ressortir son contenu informatif et de proposer une des traductions possibles.

\section{Résultats}

Traduire consiste à comprendre le contenu informatif du texte source et à le réexprimer en langue cible. La rime identifiée produit une mélodie. Celle-ci laisse entendre la voix inquiète du poète. Aussi, les autres analyses linguistiques que nous avons faites nous permettent de dire que dans le présent poème, l'auteur se lamente car l'Homme est un être prisonnier du Temps. Il appelle à la conscience de l'Homme mais surtout des plus jeunes sur la fugacité de la vie. Avec une voix d'adulte, le poète conseille aux plus jeunes de jouir de leur fraicheur car quand le corps se fait vieux, il ne peut plus ressentir les plaisirs de la vie, il développe différents maux et finit par mourir. Nous pouvons également comprendre que la voix poétique conseille de pratiquer le topic carpe diem qui est d'ailleurs une thématique anacréontique, c'est-à-dire basée sur des thèmes légers tels que le vin, l'amour (cf. Vers 1et 2 de la strophe 8), etc. L'idée générale du poème ainsi dégagée, la reformulation du texte dont la lisibilité est le critère dominant du travail accompli devient indispensable. 
Tableau 4 : Présentation du texte source et de sa traduction

\begin{tabular}{|c|c|}
\hline Texte d'origine & Texte traduit \\
\hline $\begin{array}{l}\text { A Dorila } \\
\text { S1 !Cómo se van las horas, } \\
\text { y tras ellas los días, } \\
\text { y los floridos años } \\
\text { de nuestra frágil vida! }\end{array}$ & $\begin{array}{l}\text { À Dorila } \\
\text { S1 Comme passent les heures, } \\
\text { et les jours après elles, } \\
\text { et les années fleuries } \\
\text { de notre vie fragile! }\end{array}$ \\
\hline $\begin{array}{l}\text { S2 La vejez luego viene, } \\
\text { del amor enemiga, } \\
\text { y entre fúnebres sombras } \\
\text { la muerte se avecina, }\end{array}$ & $\begin{array}{l}\text { S2 Vient ensuite la vieillesse } \\
\text { ennemie de l'amour, } \\
\text { et entre les ombres funèbres } \\
\text { s'avance la mort, }\end{array}$ \\
\hline $\begin{array}{l}\text { S3 que escuálida y temblando, } \\
\text { fea, informe, amarilla, } \\
\text { nos aterra y apaga } \\
\text { nuestros fuegos y dichas. }\end{array}$ & $\begin{array}{l}\text { S3 décharnée et tremblante, } \\
\text { hideuse, informe, jaunâtre, } \\
\text { elle nous terrifie et éteint } \\
\text { notre flamme et nos joies. }\end{array}$ \\
\hline $\begin{array}{l}\text { S4 El cuerpo se entorpece, } \\
\text { los ayes nos fatigan, } \\
\text { nos uyen los placeres } \\
\text { y deja la alegría. }\end{array}$ & $\begin{array}{l}\text { S4 Le corps s'alourdit, } \\
\text { les douleurs nous fatiguent, } \\
\text { tous les plaisirs nous fuient } \\
\text { et la joie disparaît. }\end{array}$ \\
\hline $\begin{array}{l}\text { S5 Si esto, pues, nos aguarda, } \\
\text { ¿para qué, mi Dorila, } \\
\text { son los floridos años } \\
\text { de nuestra frágil vida? }\end{array}$ & $\begin{array}{l}\text { S5 Mais, si tel est notre sort, } \\
\text { ma Dorila, à quoi servent } \\
\text { les années fleuries } \\
\text { de notre vie fragile? }\end{array}$ \\
\hline $\begin{array}{l}\text { S6 Para juegos y bailes } \\
\text { y cantares y risas } \\
\text { nos los dieron los cielos, } \\
\text { las gracias los destinan. }\end{array}$ & $\begin{array}{l}\text { S6 À jouer et danser, } \\
\text { à Chanter et à rire } \\
\text { ce sont des dons du ciel, } \\
\text { les merveilles du destin. }\end{array}$ \\
\hline $\begin{array}{l}\text { S7 Ven jay! ¿qué te detienes? } \\
\text { ven, ven, paloma mía, } \\
\text { debajo de estas parras } \\
\text { do lene el viento aspira. }\end{array}$ & $\begin{array}{l}\text { S7 Viens, pourquoi t'arrêtes-tu? } \\
\text { viens, viens ma colombe } \\
\text { sous ces treilles } \\
\text { où souffle le vent léger, }\end{array}$ \\
\hline $\begin{array}{l}\text { S8 y entre brindis süaves } \\
\text { y mimosas delicias } \\
\text { de la niñez gocemos, } \\
\text { pues vuela tan aprisa. }\end{array}$ & $\begin{array}{l}\text { S8 et par des toasts exquis } \\
\text { et des plaisirs câlins } \\
\text { jouissons de la jeunesse } \\
\text { qui trop vite s'enfuit }\end{array}$ \\
\hline
\end{tabular}

Source : Données d'une étude effectuée en 2009

Dialogos • Vol. XXII No. 38/2021 


\section{Discussion}

Selon l'organigramme du processus de traduction professionnelle élaboré par D. Gouadec (2002, p. 57), le contrôle final est l'ultime étape qu'exige cette activité. Celui-ci consiste à vérifier l'exactitude de la reformulation faite afin de s'assurer que l'équivalence rend parfaitement tout le sens de l'énoncé initial. C'est-à-dire qu'il s'agit, pour le traducteur, de vérifier «si les phrases qu'il jette sur le papier seront comprises par la collectivité linguistique à laquelle il appartient ; écrivant en français il s'assure que sa version n'est pas marquée par les formes et structure de la LO $^{9} »$ (J. Delisle (1984, p. 44-45). L'observation du texte source et la traduction qui est proposée permet de constater que, sur le plan formel, le texte traduit présente en apparence les caractéristiques du poème original. En espagnol comme en français le poème est composé de huit strophes horizontales reparties en quatrains. Le poème initial est composé de vers heptasyllabes et dans la version traduite, les vers sont pour la plupart heptasyllabes (soit un total de 11 vers et ce dans S1 v2, v4; S3 v1, v4 ; S4 v2 ; S5 v1, v4 ; S6 v2-4; S7 v1, v4). Dans le texte initial, les vers pairs 2 et 4 ont une rime assonante en í-a et les vers impairs 1 et 3 sont dépareillés. Quant au texte en français, il a été fait en vers libres avec en général des rimes masculines et féminines qui alternent entre elles (cf. S2).

En outre, comme dans le texte de départ, le poème traduit contient également des figures de rhétorique. En effet, la gradation ascendante présente en $\mathrm{S} 1$ dans le $\mathrm{TO}$ se retrouve dans la $\mathrm{S} 1 \mathrm{du}$ TA à travers les mots «heures, jours, années, vie». L'anaphore est aussi présente dans le TA. D'abord nous la remarquons dans les strophes $1 ; 2 ; 3 ; 4 ; 6 ; 8$ où elle est manifeste à travers l'emploi répété de la conjonction de coordination «et », puis nous la retrouvons dans les deux premiers vers de la S7 par le verbe conjugué "viens" et elle est doublée d'une épizeuxe en V2. Tandis que dans la S5 du texte de départ nous avons un enjambement versal en v2 et $\mathrm{v} 4$, dans le texte traduit nous obtenons un enjambement complexe, c'est-àdire composé d'un rejet et contre rejet (J. Mazaleyrat, 1974). Par ailleurs, on remarque aussi une mise en parallèle des deux premiers vers " Le corps s'alourdit (v1), les douleurs nous fatiguent (v2)» et des deux derniers « tous les plaisirs nous fuient (v4), et la joie disparaît. (v4)» en S4.

\footnotetext{
${ }^{9}$ Langue d'origine
} 
En ce qui concerne l'épithétisme présent dans le texte de départ, nous le retrouvons également dans le texte d'arrivé en S1, S2, S5 et S8 par les adjectifs qualificatifs épithètes (années fleuries; vie fragile; ombres funèbres; années fleuries; vie fragile ; toasts exquis et plaisirs câlins). Au niveau des syllabes, il est ardu d'obtenir un nombre équivalent à celui du texte source, et d'obtenir une équivalence au niveau de la rime. En effet, c'est évident car il s'agit de travailler ici sur deux langues qui obéissent chacune à des règles grammaticales qui leur sont propres. Cependant on peut remarquer que s'agissant de deux langues romanes, on retrouve des vers courts dans la version française, malgré l'impossibilité de respecter intégralement la mesure métrique. Au niveau des vers par exemple, l'heptasyllabe est un vers qui ne s'utilise presque pas dans la poésie française. Le petit Larousse 2010 souligne à cet effet que «l'heptasyllabe est rare, c'est le vers préféré de Verlaine avec l'ennéasyllabe ». Ceci permet de justifier la différence, au niveau du mètre, des vers dans les deux textes. En outre, tandis qu'en espagnol toutes les voyelles sont considérées dans le décompte des syllabes, la langue française n'obéit pas à cette règle. En effet, dans le traitement du « e » muet par exemple, B. Buffard-Moret (1997, pp. 23-24) donne ces explications :

En ancien français, toutes les voyelles écrites, y compris le e caduc, se prononcent et forment en poésie une syllabe comptable, mais le e en fin de vers n'est pas compté : c'est ce qu'on appelle l'apocope, phénomène qui s'est perpétué dans toute l'histoire de la poésie, que le e soit ou non suivi de consonnes non prononcées: Je ne vous ferai point / de reproches frivoles: Racines, Bajazet, $V, I V$.

Ces différences de règle de métrique dues à la diversité linguistique nous amènent à comprendre que le TS et le TC ne peuvent pas être équivalents tant au niveau du mètre qu'au niveau de la rime. Nous sommes en effet partie d'un texte heptasyllabe (TO) pour aboutir à un texte dont la plupart des vers sont hexasyllabes, (soit dix-huit (18) vers hexasyllabes (en TA) sur un total de trente-deux (32) vers), c'est-à-dire que le TC est majoritairement composé de démis-alexandrins. Nous pensons que la perte de syllabes en TC due à l'e muet en fin de vers serait une cause importante de ce résultat. Aussi, selon la tradition de la langue française, « les vers de six syllabes (6) la plupart du temps sont employés dans des compositions où ils figurent avec des vers plus longs. Toutefois, l'hexasyllabe a dû être employé seul dans des chansons », (M. Grammont 
1976, p. 45). En effet, cette particularité peut amener à envisager ce vers comme propice à évoquer des états intérieurs (joie, tristesse, mélancolie, prière...) qui sont le propre du registre de la chanson et principalement de l'ode que Le Robert définit comme un poème lyrique destiné à être chanté. De fait, le registre fonctionnel de l'ode pourrait être avantagé dans la version traduite en français et donc, d'une langue à l'autre, le poème garde sa fonction, celle d'être un texte sur fond de chanson, donc un chant poétisé.

Cette réflexion montre à quel point l'exploitation du texte poétique au cours de la traduction pédagogique peut être laborieuse mais bénéfique aux apprenants. L'étude permet d'édifier sur le caractère interdisciplinaire et transdisciplinaire de l'activité de traduction scolaire, laquelle conduit indéniablement à explorer le monde de la métrique souvent exploité en classe de littérature (option poésie). L'étude des éléments métriques du poème peut inviter l'apprenant à comprendre certains faits. D'abord, le texte littéraire n'est pas soumis à une exhaustivité interprétative. Ensuite, l'analyse du poème, texte pris dans son entièreté ${ }^{10}$, semble plus exigeante par opposition à un texte narratif dont l'examen reposerait seulement sur les éléments linguistiques (syntaxe, sémantique, rhétorique, lexique) et extralinguistique. En effet, en plus des éléments linguistiques à analyser (J.C. Gémar, 1995), l'apprenant sera invité à activer son bagage cognitif et à affiner son ouïe afin de découvrir et d'apprécier la subtilité et le génie de chaque langue. L'on comprend aisément que le poème, en tant que support de traduction, est un texte complet qui permet la manipulation d'une pléthore d'outils pour en saisir le sens. L'analyse axée sur la métrique devient importante dans la mesure où elle vient en renforcement et ou en substitution aux cours de littératures (option poésie) reçus ou pas en parallèle. En renforcement, l'étude donnera la possibilité à l'apprenant de combler les éventuelles lacunes qu'il a sur le point en exergue. En substitution, l'étude joue un rôle double. C'est-à-dire qu'en plus d'être un cours de traduction, il est un cours de lecture du poème en vers et les outils convoqués concourent à une évidence: l'apprentissage et le perfectionnement des langues en présence que sont l'espagnol et le français et le développement des compétences métatextuelles (I. Duval, 2004).

10 En classe de traduction pédagogique, les autres textes littéraires sont généralement des extraits de textes, donc offrant juste une infime partie du contenu de l'œuvre dont il est tiré. 


\section{Conclusion}

En définitive, l'on peut dire que l'enseignement de la traduction de la poésie revêt un intérêt pratique considérable pour les formateurs issus de l'ENS. Avec la clé des mots et des images métaphorisées du poète, il ouvre à l'altérité, c'est-à-dire à la connaissance de l'univers philosophique, linguistique et ontologique de l'Autre - le poète - pour saisir le sens de ses mots/maux, de ses émotions les plus intimes, d'appréhender sa vision du monde. Sur le plan de sa structure interne, les différentes analyses convoquées montrent que le poème ne révèle sa splendeur qu'au prix de la connaissance des unités techniques qui font sa singularité genrologique. Alors, on aura très bien compris que c'est précisément la maîtrise de ces dernières, le processus même d'appropriation des mots du poète, en dépit de la complexité ainsi relevée, qui permet de s'ouvrir à la connaissance de l'Autre et, par conséquent, à la connaissance de soi-même. Sur un plan purement pédagogique, la traduction du poème permet le développement des compétences métatextuelles et des compétences en écriture. Ainsi, exclure la poésie du champ des enseignements de traduction dans une institution ayant vocation de former des formateurs en langues, c'est produire, à la fin, des professionnels unijambistes avec des profils lacunaires qu'ils reproduiront indéfiniment. La prise en compte de la pédagogie du transfert du texte poétique permettrait de retrouver l'équilibre qui, jusque-là fait défaut dans les programmes de formation du département d'espagnol de l'ENS. C'est à la réalisation de ce vœu pieux que nous appelons par cette contribution dont la description d'une expérimentation pratique de classe constituera une réflexion subséquente.

\section{Bibliographie}

1. BACRY Patrick, 1992, Les figures de style, Paris, Belin

2. BONNEFOY Yves, 2000, La Communauté des traducteurs, Strasbourg, Presses universitaires de Strasbourg.

3. BUFFARD-MORET Brigitte, 1997, Introduction à la versification, Paris, Dunod.

4. CHEVALIER Jean et CHEERBRANT Alain, 1982, Dictionnaire des symboles, Paris, Jupiter.

5. DUVAL Isabelle, 2004, «La poésie et l'enseignement du français » Québec français, (135), pp. 42-45. 
6. ELLRODT Robert, 2006, «Comment traduire la poésie ?», Palimpsestes [en ligne], Hors-série, pp. 65-75, http://palimpsestes.revues.org/247, consulté le 30 octobre 2019.

7. FLEURBAEY Marc, BLANCHET Didier, 2013, Beyond GDP: Measuring Welfare and Assessing Sustainability, Oxford University Press.

8. FONTANIER Pierre, 1968, Les figures du discours, Paris, Flammarion.

9. GEMAR Jean- Claude, 1995, Traduire ou l'art d'interpréter : fonctions, statut et esthétique de la traduction, Tome 1 : principes, Québec, Presses universitaires de Québec.

10. GOUADEC Daniel, 2002, Profession: Traducteur, Paris, La maison du Dictionnaire.

11. GRAMMONT Maurice, 1976, Petit traité de versification française, Paris, Armand Colin.

12. GREVISSE Maurice, 2000, Précis de grammaire française, Bruxelles, Duculot.

13. 2008, Le bon usage, Bruxelles, De Boeck.

14. LECLERCQ Guy, 2001, "Lecture descriptive pour la traduction de « la musique » un sonnet des Fleurs du mal de Baudelaire », Traduire 190-191, la traduction littéraire 1ère partie, pp.85-115.

15. MAZALEYRAT Jean, 1974, Eléments de métrique française, Paris, A. Colin.

16. PARDO Madeleine et PARDO Arcadio, 2007, Précis métrique espagnole, Paris, Armand Colin.

17. QUILIS Antonio, 1999, Métrica española, Barcelona, Ariel. 